\title{
Modeling, Control and Simulation of a Novel Mobile Robotic System
}

\author{
Xiaoli Bai, Jeremy Davis, James Doebbler, James D.Turner and John L.Junkins*
}

\begin{abstract}
We are developing an autonomous mobile robotic system to emulate six degree of freedom relative spacecraft motion during proximity operations. A mobile omni-directional base robot provides $\mathrm{x}, \mathrm{y}$, and yaw planar motion with moderate accuracy through six independently driven motors. With a six degree of freedom micro-positioning Stewart platform on top of the moving base, six degree of freedom spacecraft motion can be emulated with high accuracy. This paper presents our approach to dynamic modeling, control, and simulation for the overall system. Compared with other simulations that introduced significant simplifications, we believe that our rigorous modeling approach is crucial for the high fidelity hardware in-the-loop emulation.
\end{abstract}

Keywords: Dynamic Modeling, Non-Linear Control, System Simulation

\section{Introduction}

We in the Department of Aerospace Engineering at Texas A\&M University are developing an autonomous mobile robotic system to emulate six degree of freedom (DOF) relative spacecraft motion during proximity operations [1], [2]. The base uses an active split offset castor (ASOC) drive train to achieve omni-directional planar motion with desired tracking position errors in the $\pm 1 \mathrm{~cm}$ range and heading angle error in the $\pm 0.5^{\circ}$ range. With six independently controlled wheels, we achieve a nominally uniform motor torque distribution and reduce the total disturbances with system control redundancy. A CAD (Computer-aided Design) sketch of our one-third scale model prototype is shown in Fig. 1.

A micro-positioning Stewart platform is mounted on the full-size moving base, as shown in Fig. 2. For the Stewart platform, the base plate and top plate are connected by six extensible legs; the parallel nature provides higher stiffness, higher loading capacities, and higher frequency compared with typical serial positioning devices. A complete dynamic model and a robust adaptive controller for the Stewart platform have been developed using a novel automatic differentiation method in [3] and [4]. The novel modeling approach makes it easy to modify the model assumptions and eliminate complicated derivative calcu-

*The authors are with the Department of Aerospace Engineering, Texas A\&M University, College Station, TX 77843 (email: xiaolibai@tamu.edu; jeremy.davis@tamu.edu; james.doebbler@tamu.edu; turner@aeromail.tamu.edu; junkins@tamu.edu)

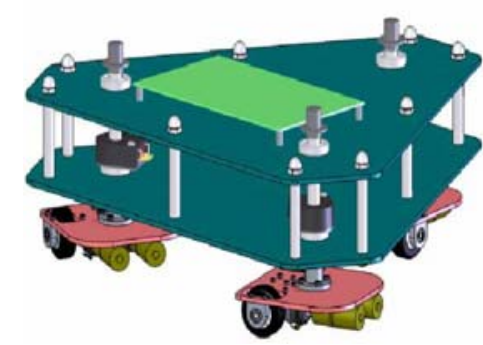

Figure 1: Base robot prototype

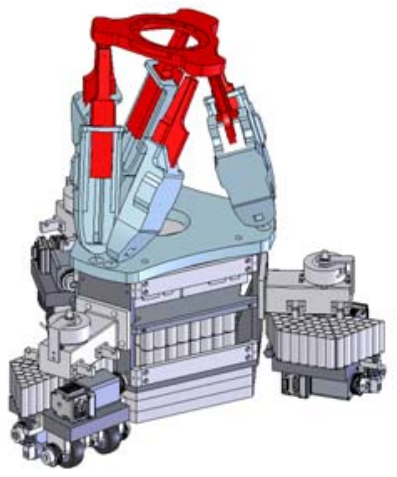

Figure 2: Platform on the base

lations. The robust adaptive control law guarantees that the tracking errors are asymptotically stable under even large parameter errors and slow-changing or constant external disturbances.

This paper focuses on modeling and control issues for the mobile base as well as the overall system simulation. Section 2 presents a dynamic model for the mobile base using a Lagrangian approach. Two control laws for the base are compared. One uses an input-output feedback method to design a dynamic control law. The other uses a kinematic control method. In Section 3, the overall system simulation approach is described and the simulation results are shown. Conclusions are presented in Section 4. 


\section{Dynamics and Control of the Moving Base}

Using ASOCs to provide true omni-directional mobility was introduced by H.Yu [5]. While kinematics and implementation have been discussed in [5], no dynamic models have been studied for mobile robots driven by ASOCs. In fact, there have been many papers written about solving the problem of dynamic model formulation under nonholonomic constraints using the kinematic model, but only a few papers addressing the integration of the nonholonomic kinematic controller with the dynamics of the mobile robot [6]. "Perfect velocity tracking" is always assumed in the available papers, which may not be the truth in reality [7]. This chapter formulates a rigorous approach to track the reference path while taking account of the system dynamics. The mobile base we propose in this chapter not only achieves true omni-directional motion without wheel reorientation, but also achieves higher precision control with higher loading capacity compared with the models discussed in [6], [7], [8]. Furthermore, our modeling and control approaches are very general and applicable to a significant class of similar systems.

\subsection{Kinematic Equations}

A top view representation of the entire base assembly with the frame definitions is shown in Fig. 3. The three vertex points of the triangular base are all pivot points, each of which is connected to a castor. The castor is connected to two wheels through shafts. Each castor is free to rotate about its pivot point and each wheel is independently driven by a mounted motor.

Kinematic equations are derived as follows. Knowing the velocity of the mass center of the triangular base together with the measured castor angles, and utilizing non-slippage constraints for the six wheels (the wheels roll without slip and also can not have side slip), the velocity of each wheel and shaft is uniquely defined. The velocity of the triangular base includes its translational velocity $\mathbf{v}_{b}=[\dot{x}, \dot{y}, 0]^{\top}$ and rotational velocity $\dot{\psi}$ about its center of mass, where $x$ and $y$ are the base positions in the inertial frame and $\psi$ is the rotational angle between the base body frame and the inertial frame.

The velocity of the $i^{\text {th }}$ pivot point is computed by

$$
\mathbf{v}_{p_{i}}=\mathbf{v}_{b}+\mathbf{B I}\left[-\dot{\psi} R \sin \left(\phi_{i}\right), \dot{\psi} R \cos \left(\phi_{i}\right), 0\right]^{\top}
$$

where $\phi$ is the angle between $\hat{\mathbf{b}}_{1}$ and the line connecting base center of mass to the $i^{\text {th }}$ pivot point, and for the symmetric base, $\phi_{1}=0^{\circ}, \phi_{2}=120^{\circ}$, and $\phi_{3}=240^{\circ}$; BI is the direction cosine matrix that transforms components of a vector in the $\mathbf{B}$ frame $\left\{\hat{\mathbf{b}}_{1}, \hat{\mathbf{b}}_{2}, \hat{\mathbf{b}}_{3}\right\}$ to the same vector with components in the inertial frame with the form

$$
\mathbf{B I}=\left[\begin{array}{ccc}
\cos (\psi) & -\sin (\psi) & 0 \\
\sin (\psi) & \cos (\psi) & 0 \\
0 & 0 & 1
\end{array}\right]
$$

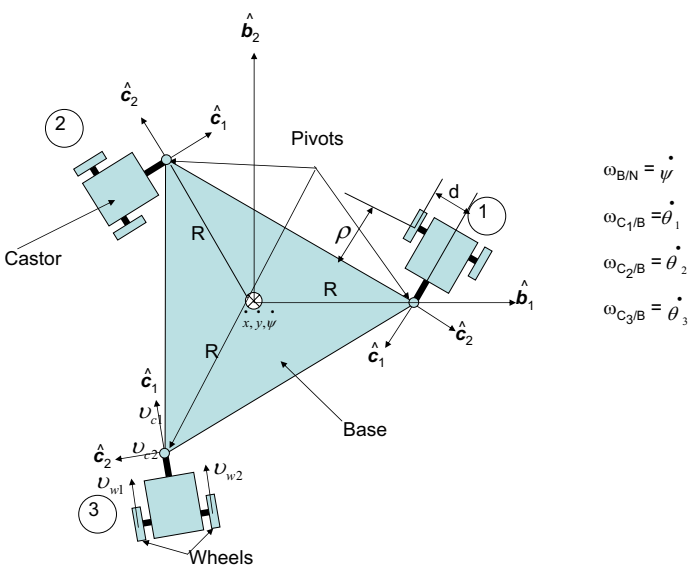

Figure 3: Top view of the base

$R$ is the distance from the base center of the mass to the pivot point. The inertial velocity of the pivot point is expressed in the castor $\mathbf{C}$ frame $\left\{\hat{\mathbf{c}}_{1}, \hat{\mathbf{c}}_{2}, \hat{\mathbf{c}}_{3}\right\}$ as $\mathbf{v}_{c_{i}}=$ $\left[v_{c_{1}}, v_{c_{2}}, 0\right]$ using

$$
\mathbf{v}_{c_{i}}=\mathbf{I C} \mathbf{v}_{p_{i}}
$$

where

$$
\mathbf{I C}=\left[\begin{array}{ccc}
\cos \left(\psi+\theta_{i}\right) & \sin \left(\psi+\theta_{i}\right) & 0 \\
-\sin \left(\psi+\theta_{i}\right) & \cos \left(\psi+\theta_{i}\right) & 0 \\
0 & 0 & 1
\end{array}\right]
$$

and $\theta_{i}$ is the rotational angle between the $i^{\text {th }}$ castor $\mathbf{C}$ frame and the base $\mathbf{B}$ frame. Angular velocity of the castor with respect to the inertial frame is

$$
\omega_{i}=\dot{\theta}_{i}+\dot{\psi}
$$

Using the no side slip constraints, the angular velocity can also be expressed as

$$
\omega_{i}=v_{c_{2}} / \rho
$$

where $\rho$ is the distance from the pivot point to the center of the wheels along $\hat{\mathbf{c}}_{1}$ direction.

The velocities of the two wheels are

$$
\begin{aligned}
& v_{w_{1}}=v_{c_{1}}-d \omega_{i} \\
& v_{w_{2}}=v_{c_{1}}+d \omega_{i}
\end{aligned}
$$

where $d$ is the distance from the pivot point to the center of the wheels along $\hat{\mathbf{c}}_{2}$ direction. The velocities of the shafts are solved for in the same way.

\subsection{Dynamic Equations}

For the mobile base, the system energy includes the kinetic energy of the triangular base, three castors, six shafts and six wheels. Since we ignore the vertical 
motions in this paper, no potential energy is involved. The same approach developed below will be used in the future to derive a three dimensional model, which includes the potential energy change because of the floor irregularities.

Twelve generalized coordinates $\boldsymbol{q}=$ $\left[x, y, \psi, \theta_{1}, \theta_{2}, \theta_{3}, \Pi_{1}, \Pi_{2}, \Pi_{3}, \Pi_{4}, \Pi_{5}, \Pi_{6}\right]^{T} \quad$ are chosen for the base system, which includes two translational and one angular position of the triangular base, three angular positions of the castors, and six angular positions of the wheels, respectively. The corresponding twelve generalized velocities are not independent. Firstly, since Eqs. 5 and 6 describe the same quantity, we have the first set of constraints

$$
w_{i}-v_{c_{2}} / \rho=0
$$

Secondly, using the rolling without slip constraints for the wheels, we have

$$
\begin{aligned}
& R_{w} \dot{\Pi}_{1}-v_{w_{1}}=0 \\
& R_{w} \dot{\Pi}_{2}-v_{w_{2}}=0
\end{aligned}
$$

Eqs. 12, 13, and 14 provide three constraints for each set of the castors, leading to nine kinematic constraints in total. Since these non-slippage constraints are nonholonomic, we need to use Lagrangian multipliers to formulate the equations of motion (EOM) using twelve coordinates with nine constraint equations without reducing the system to minimal order by substituting the constrained relationships.

\subsection{Dynamics and Control Law}

Since we ignore the system small vertical motions in this chapter, no potential energy is involved. The energy of the mobile base is the summation of the kinetic energy of the triangular base, three castors, six shafts and six wheels. The same approach developed below will be used in the future to derive a three dimensional model, which includes the potential energy change because of the floor irregularities.

Twelve generalized coordinates

$$
\mathbf{q}=\left[x, y, \psi, \theta_{1}, \theta_{2}, \theta_{3}, \Pi_{1}, \Pi_{2}, \Pi_{3}, \Pi_{4}, \Pi_{5}, \Pi_{6}\right]^{\top}
$$

are chosen for the base system, which includes two translational and one angular position of the triangular base, three angular positions of the castors, and six angular positions of the wheels, respectively. The corresponding twelve generalized velocities are not independent. Firstly, since Eqs. 5 and 6 describe the same quantity, we have the first set of constraints

$$
w_{i}-v_{c_{2}} / \rho=0
$$

Secondly, using the rolling without slip constraints for the wheels, we have

$$
\begin{aligned}
& R_{w} \dot{\Pi}_{1}-v_{w_{1}}=0 \\
& R_{w} \dot{\Pi}_{2}-v_{w_{2}}=0
\end{aligned}
$$

where $R_{w}$ is the radius of the wheels. Equations 12,13 and 14 provide three constraints for each castor, leading to nine kinematic constraints in total. Since these non-slippage constraints are non-holonomic, we need to use Lagrangian multipliers to formulate the equations of motion (EOM) using twelve coordinates with nine constraints, while not trying to reduce the system to a minimal order representation by substituting the constraint relationships.

The final EOM is formulated as

$$
M(\mathbf{q}) \ddot{\mathbf{q}}+N(\mathbf{q}, \dot{\mathbf{q}}) \dot{\mathbf{q}}+G(\mathbf{q})=B(\mathbf{q}) \mathbf{u}+C^{\top} \boldsymbol{\lambda}
$$

The kinematic constraints are not dependent on time and can be expressed as

$$
C \dot{\mathbf{q}}=0
$$

where $\dot{\mathbf{q}}$ is the generalized velocity and $M(\mathbf{q})$ is the $12 \times 12$ symmetric, bounded, positive definite mass matrix; $N(\mathbf{q}, \dot{\mathbf{q}}) \dot{\mathbf{q}}$ represents the centripetal and Coriolis torque, $G(\mathbf{q})$ is the gravity torque, $B(\mathbf{q})$ is the matrix that transforms the input $\mathbf{u}$ to the generalized force, $C$ is the $9 \times 12$ constraint matrix, $\boldsymbol{\lambda}$ is the Lagrangian multiplier, and $C^{T} \boldsymbol{\lambda}$ is the constraint force. It has been proven that these kind of non-holonomic dynamic systems can not achieve asymptotical stability using smooth time-invariant state feedback [9]. The stabilization methods proposed so far include discontinuous time-invariant stabilization, time-varying stabilization, and hybrid feedback [10].

To simplify the controller for real-time application, null space formulation is used to find a solution [8]. Choose $S(\mathbf{q})$ to be a $12 \times 3$ matrix, which is formed by three smooth and linearly independent vector fields spanning the null space of $C$, leading to

$$
S^{\top} C^{\top}=0
$$

According to Eqs. 16 and 17, since the constrained generalized velocity is always in the null space of $C$ which is characterized by Eq. 16, a vector $\mathbf{v}(t) \in R^{3}$ can be constructed such that

$$
\dot{\mathbf{q}}=S(\mathbf{q}) \mathbf{v}
$$

Notice that the choice of $S$ and $\mathbf{v}$ is not unique. In general, $\mathbf{v}$ is an abstract variable and may not have any physical meaning.

Differentiating Eq. 18, substituting it into Eq. 15, and pre-multiplying the resulted equation by $S^{\top}$, we get the transformed EOM

$$
S^{\top} M S \dot{\mathbf{v}}+\left(S^{\top} M \dot{S}+S^{\top} N S\right) \mathbf{v}+S^{\top} G=S^{\top} B \mathbf{u}
$$

The new system state space equation is

$$
\dot{\mathbf{x}}=\left[\begin{array}{c}
S \mathbf{v} \\
\mathbf{0}
\end{array}\right]+\left[\begin{array}{l}
0 \\
I
\end{array}\right] \tau
$$


when the state-space vector is chosen as $\mathbf{x}=\left[\mathbf{q}^{\top}, \mathbf{v}^{\top}\right]^{\top}$, and the control input is

$$
\tau=\left(S^{\top} M S\right)^{-1}\left(S^{\top} B \mathbf{u}-\left(S^{\top} M \dot{S}+S^{\top} N S\right) \mathbf{v}-S^{\top} G\right) .
$$

Since for our 6-DOF motion emulation, the requirement for the mobile base robot is to provide a satisfactory trajectory that can extend the planar workspace of the upper high precision Stewart platform, a Lyapunov method is used to design such an input-output feedback control law that guarantees the position and orientation errors of the mobile base are asymptotically stable and is simpler to apply than either discontinuous or time-varying control. The output equation is defined as

$$
\mathbf{Y}=h(\mathbf{q})=[x ; y ; \psi]
$$

Using Eq. 18, the output velocity equation is expressed as

$$
\dot{\mathbf{Y}}=\frac{\partial h}{\partial \mathbf{q}} S \mathbf{v}=J S \mathbf{v}=\phi \mathbf{v}
$$

where $J=\frac{\partial h}{\partial \mathbf{q}}$, and $\phi$ is the decoupling matrix. The necessary and sufficient condition for the input-output linearization is that the decoupling matrix has full rank. For our case, the determinant of $\phi$ is 1 , thus it never becomes singular. Using the Lyapunov method, we design a control law for $\tau$ to track reference position $\mathbf{Y}_{r}(t)$ and reference velocity $\dot{\mathbf{Y}}_{r}(t)$. The Lyapunov function is defined as

$$
V=1 / 2\left(\mathbf{Y}_{r}-\mathbf{Y}\right)^{\top} K_{1}\left(\mathbf{Y}_{r}-\mathbf{Y}\right)+1 / 2\left(\dot{\mathbf{Y}}_{r}-\dot{\mathbf{Y}}\right)^{\top} K_{2}\left(\dot{\mathbf{Y}}_{r}-\dot{\mathbf{Y}}\right)
$$

It is easy to prove that using the control law in Eq. 25, the output tracking errors are asymptotically stable

$$
\begin{array}{r}
\tau=(J S)^{-1}\left(\ddot{\mathbf{Y}}_{r}+K_{1} \mathbf{e}+K_{2} \dot{\mathbf{e}}-J \dot{S} \mathbf{v}+K_{3} \epsilon\right) \\
\mathbf{e}=Y_{r}-Y \\
\dot{\mathbf{e}}=\dot{Y}_{r}-\dot{Y} \\
\dot{\epsilon}=\mathbf{e}
\end{array}
$$

The input torques from the six motors are solved for using Eq. 21. Notice that $B$ is a $12 \times 6$ matrix for this redundantly controlled system. The pseudoinverse is used for the inverse of $S^{\top} B$, yielding a minimal motor control effort.

\subsection{Simulation Results}

Firstly, as one of the criteria to validate the dynamic model, we numerically solve the EOM when there is no external input and then check whether the system kinematic energy remains constant or not. Theoretically, the energy should be constant. To avoid the constraint drift during integration, we utilize a constraint stabilization method which was first proposed in [11]. Dynamic response when the center of the base is doing a pure rotating motion has been validated through the simulation.
The relative errors for the energy change $\delta_{e}$, no side slip constraint $\delta_{p r}$ and rolling without slip constraint $\delta_{n s}$, are calculated as follows and are shown in Figs. 4, 5, and 6 .

$$
\begin{array}{r}
\delta_{e}=\left(E(t)-E\left(t_{0}\right)\right) / E\left(t_{0}\right) \\
\delta_{p r}=\left(C_{p r}(t)-C_{p r}\left(t_{0}\right)\right) / C_{p r}\left(t_{0}\right) \\
\delta_{n s}=\left(C_{n s}(t)-C_{n s}\left(t_{0}\right)\right) / C_{n s}\left(t_{0}\right)
\end{array}
$$

where $E(t)$ is the system energy at time $t, C_{p r}(t)=\dot{\theta}_{1}+$ $\dot{\psi}-v_{c_{2}} / \rho$ is the first castor no side slip constraint defined in Eq. 12, and $C_{n s}(t)=R_{w} \dot{\Pi}_{1}-v_{w_{1}}$ is the first wheel rolling without slip constraint defined in Eq. 13.

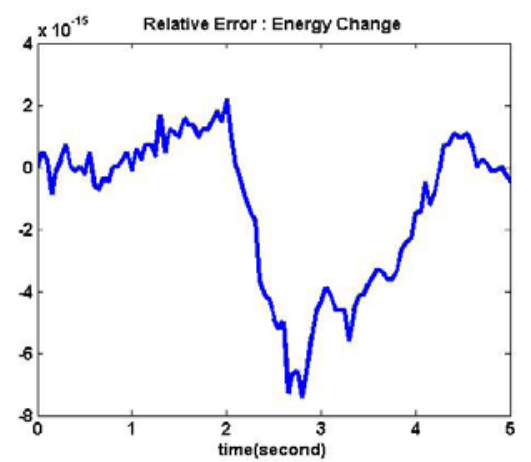

Figure 4: Energy variation history

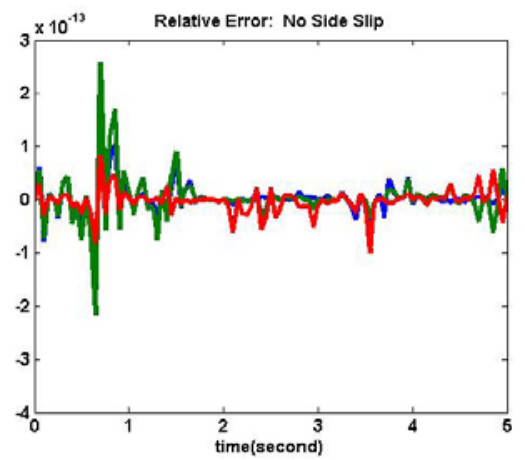

Figure 5: No side slip constraint

Secondly, the mobile base is commanded to track a constant velocity reference trajectory with initial position and velocity errors with all the wheels starting in a pulling position. We compare the tracking results using the dynamic controller proposed in Eq. 25 with a kinematic controller.

For the kinematic controller, knowing the reference trajectory of the mass center of the base, the top level controller generates a commanded base velocity $\mathbf{V}_{c}=$ $\dot{\mathbf{Y}}_{r}-K\left(\mathbf{Y}-\mathbf{Y}_{r}\right)$, where all the symbols are defined as in Eq. 24. Through the unique mapping from the base center velocities to the wheel velocities, the kinematic controller commands the motors to track the commanded 


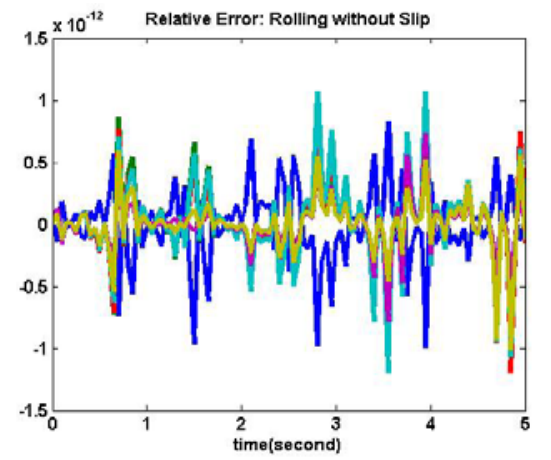

Figure 6: Rolling without slip constraint

wheel velocities.

Parameters of Animatics SmartMotor 2315DT [12] are utilized in the simulation. For the dynamic controller, we achieve the commanded torque through commanding a corresponding quantized motor input voltage. The kinematic controller runs through a PID loop internal to each motor to achieve the commanded velocity.

Simulation results are shown in Figs. 7, 8, and 9. We find that both controllers can achieve satisfactory tracking while the dynamic response can be changed through tuning gains. In addition, the dynamic controller is more efficient than the kinematic controller in terms of the control efforts involved, although the dynamic controller needs more computation time than the kinematic controller. For the kinematic controller, since each wheel does not have any information about other wheels, they may fight each other during the motion. We point out that no friction models are implemented in these simulations.
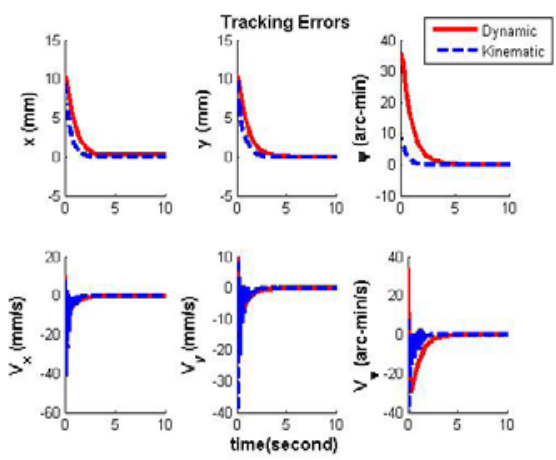

Figure 7: Tracking errors comparison

\section{Overall System Simulation}

\subsection{System Equations of Motion}

Mobile manipulators have received significantly increased interest in the industrial, military, and public communi-
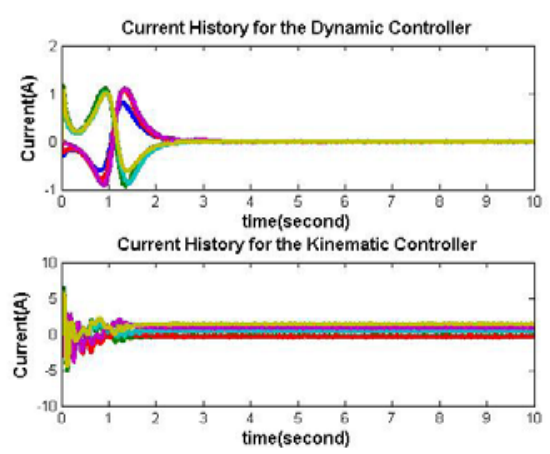

Figure 8: Current comparison
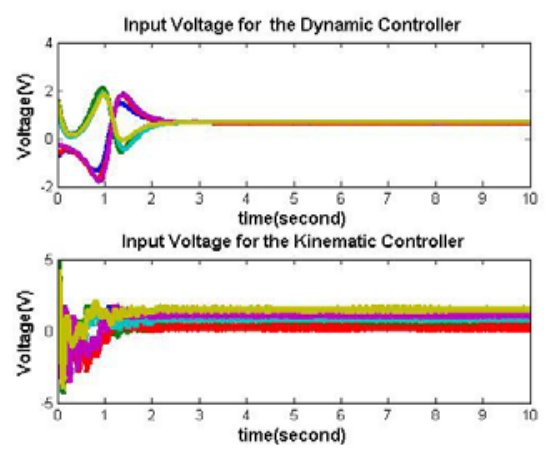

Figure 9: Voltage comparison

ties for their mobility combined with the manipulator's dexterous abilities. But most current work treats the system without considering dynamic interactions, and only copes with holonomic constraints, or just considers the kinematic interactions [13], [14]. Since our final aim is to do a high fidelity emulation, the previous simplifications are not acceptable to us at the dynamic modeling level. We formulate a complete model of the Stewart platform and a mobile base separately at first. For each subsystem, a robust controller is designed to account for the dynamic interaction forces. All the generalized coordinates with the dynamic interaction forces are solved simultaneously to generate the true system dynamic response.

For the 6-DOF Stewart platform system, we choose the mass center location $\mathbf{R}_{c}=\left[X_{m}, Y_{m}, Z_{m}\right]^{\top}$, three Euler angles $\theta_{m}=\left[\theta_{1_{m}}, \theta_{2_{m}}, \theta_{3_{m}}\right]$ of the top plate, and the position and orientation of the base plate $\mathbf{q}_{b_{m}}=[x, y, \psi]^{\top}$ as the generalized coordinates. Let

$\mathbf{q}_{m}=\left[X_{m}, Y_{m}, Z_{m}, \theta_{1_{m}}, \theta_{2_{m}}, \theta_{3_{m}}\right]^{\top}$. Note that all of these coordinates are expressed with respect to the inertial frame. The moving base is modeled using twelve generalized coordinates

$\mathbf{q}_{b}=\left[x_{b}, y_{b}, \psi_{b}, \theta_{1}, \theta_{2}, \theta_{3}, \Pi_{1}, \Pi_{2}, \Pi_{3}, \Pi_{4}, \Pi_{5}, \Pi_{6}\right]^{\top}$. Let

$\begin{array}{lll}\mathbf{q}_{\bar{b}} \quad\left[x_{b}, y_{b}, \psi_{b}\right]^{\top} & \text { and } \mathbf{q}_{\bar{o}} & =\end{array}$ $\left[\theta_{1}, \theta_{2}, \theta_{3}, \Pi_{1}, \Pi_{2}, \Pi_{3}, \Pi_{4}, \Pi_{5}, \Pi_{6}\right]^{\top}$. Since the base 
plate of the Stewart platform and the triangular base of the mobile robot are rigidly connected, we assume that $\mathbf{q}_{\bar{b}}=\mathbf{q}_{b_{m}}$. Thus for the Stewart platform, the dynamic equations are expressed as

$\left[\begin{array}{cc}M_{1_{m}} & M_{12_{m}} \\ M_{12_{m}}^{\top} & M_{2_{m}}\end{array}\right]\left(\begin{array}{c}\ddot{\mathbf{q}}_{m} \\ \ddot{\mathbf{q}}_{\bar{b}}\end{array}\right)+\left[\begin{array}{c}N_{1} \\ N_{2}\end{array}\right]=\left[\begin{array}{c}Q_{1_{l}} \\ Q_{2_{l}}\end{array}\right]+\left[\begin{array}{c}0_{6 \times 6} \\ Q_{d}\end{array}\right]$

where the mass matrix of the Stewart platform $M_{m}$ is

$$
M_{m}=\left[\begin{array}{cc}
M_{1_{m}} & M_{12_{m}} \\
M_{12_{m}}^{\top} & M_{2_{m}}
\end{array}\right]
$$

$Q_{1}$ and $Q_{2}$ are the generalized forces generated by the Stewart platform actuator motors and projected on the generalized coordinates $\mathbf{q}_{m}$ and $\mathbf{q}_{\bar{b}}, Q_{d}$ are the interaction forces/torques projected on the $\mathbf{q}_{\bar{b}}$ coordinates, and $N_{1}$ and $N_{2}$ are other nonlinear terms. Details about solving for the compact form of the mass matrix $M$ can be found in [3], [4], and [15].

For the mobile moving base, the final organized dynamic equations, which have the Lagrangian multipliers eliminated by taking the derivatives of Eq. 16 and substituting Eq. 15 into them, are

$$
\begin{aligned}
& {\left[\begin{array}{cc}
M_{1_{b}} & M_{12_{b}} \\
M_{12_{b}}^{\top} & M_{2_{b}}
\end{array}\right]\left(\begin{array}{c}
\ddot{\mathbf{q}}_{\bar{b}} \\
\ddot{\mathbf{q}}_{\bar{o}}
\end{array}\right)+\left[\begin{array}{c}
N_{1_{b}} \\
N_{2_{b}}
\end{array}\right]=\left[\begin{array}{l}
Q_{1_{b}} \\
Q_{2_{b}}
\end{array}\right]} \\
& +\left[\begin{array}{c}
K_{1} Q_{d} \\
K_{2} Q_{d}
\end{array}\right]
\end{aligned}
$$

where the mass matrix of the mobile base $M_{b}$ is

$$
M_{b}=\left[\begin{array}{cc}
M_{1_{b}} & M_{12_{b}} \\
M_{12_{b}}^{\top} & M_{2_{b}}
\end{array}\right]
$$

In Eq. 31

$$
\begin{array}{r}
K=C^{\top}\left(C M_{b}^{-1} C^{\top}\right)^{-1} C M_{b}^{-1}-I \\
=\left[\begin{array}{cc}
K_{1} & K_{3} \\
K_{2} & K_{4}
\end{array}\right] \\
Q_{1 b}=\left[\begin{array}{ll}
K_{1} & K_{3}
\end{array}\right] Q_{t} \\
Q_{2 b}=\left[\begin{array}{ll}
K_{2} & K_{4}
\end{array}\right] Q_{t}
\end{array}
$$

$K_{1}$ is a $9 \times 3$ matrix, $K_{2}$ is a $3 \times 3$ matrix, $K_{3}$ is a $9 \times 9$ matrix, and $K_{4}$ is a $3 \times 9$ matrix; $Q_{t}$ are the generalized forces resulting from the base motors; $\mathrm{C}$ is the constraint matrix; $\mathrm{I}$ is the $12 \times 12$ identity matrix; $N_{1_{b}}$ and $N_{2_{b}}$ are the remaining nonlinear terms. Equations 29 and 31 can be reformulated as

$$
M_{t}\left[\begin{array}{c}
\ddot{\mathbf{q}_{m}} \\
\ddot{\mathbf{q}_{\bar{b}}} \\
\ddot{\mathbf{q}_{\bar{o}}} \\
Q_{d}
\end{array}\right]+\left[\begin{array}{c}
N_{1} \\
N_{2} \\
N_{1_{b}} \\
N_{2_{b}}
\end{array}\right]=\left[\begin{array}{c}
Q_{1_{l}} \\
Q_{2_{l}} \\
Q_{1_{b}} \\
Q_{2_{b}}
\end{array}\right]
$$

where

$$
M_{t}=\left[\begin{array}{cccc}
M_{1_{m}} & M_{12_{m}} & 0_{6 \times 9} & 0_{6 \times 3} \\
M_{12_{m}}^{\top} & M_{2_{m}} & 0_{3 \times 9} & -I_{3 \times 3} \\
0_{9 \times 6} & M_{1_{b}} & M_{12_{b}} & -K_{1} \\
0_{3 \times 6} & M_{12_{b}}^{\top} & M_{2_{b}} & -K_{2}
\end{array}\right]
$$

Equation 37 is the final form to generate the overall system dynamic response.

\subsection{System Simulation Results}

A tracking scenario is designed as

$$
\begin{aligned}
x_{b} & =0.1 t \\
x_{s}=10^{-3} \sin \left(\frac{2 \Pi}{0.1} t\right) & +0.1 t
\end{aligned}
$$

where $x_{b}$ is the reference tracking position for the base robot along $x$ direction; $x_{s}$ is the reference tracking position for the Stewart platform along $x$ direction; and the commanded motions along all the other directions for both the base robot and the Stewart platform are zero. In the simulation, the bearing frictions and scrubbing torques are included in the dynamic model as the disturbances. The bearing frictions $M_{b}$ are assumed to have the form

$$
M_{b}=-c_{f} \dot{q}
$$

where $c_{f}$ is the bearing friction coefficient obtained from experimental data and $\dot{q}$ is the wheel velocity. Usually the scrubbing torque is defined as the torque needed to twist a single wheel around its vertical axis [5]. We use a simple way to calculate this torque $M_{s}$ according to a linear form

$$
M_{s}=k r+b
$$

where rotation radius $r$ is the distance from the center of the rotation of the two wheels to the individual wheel, $k$ and $b$ are the coefficients obtained by fitting experimental data using the linear equation assumption in Eq. 42. We also assume that if the rotation radius is larger than some limit, the scrubbing torque will be zero.

Simulation results are shown in Figs 10, 11, and 12 with both the Stewart platform control frequency and the base robot control frequency chosen as $1000 \mathrm{~Hz}$. The position errors and velocity errors satisfy the usual spacecraft rendezvous and docking motion requirements [1], and we validate our control methodology when the frequency of the Stewart platform becomes as high as $10 \mathrm{~Hz}$. We point out that when the Stewart platform motion is slow, we can achieve the satisfactory tracking with lower control frequency, while when the Stewart platform frequency increases, the tracking performance gets worse. A more advanced robust control method needs to be designed in the near future to counteract the higher frequency constraint forces, which result from the faster relative motion and are treated as unknown disturbances in the control law design. In addition, since we already have equations of motion for the overall system, we may also design a control law based on the total system dynamic information. Although this control law automatically takes into account the constraint forces effect, it is very complicated and difficult to be solved. We believe our methodology to design robust control law for each subsystem in an uncoupled fashion is easier to implement, and also more robust with respect to system uncertainties, compared with designing a gigantic coupled control law based on the full system dynamic information. 

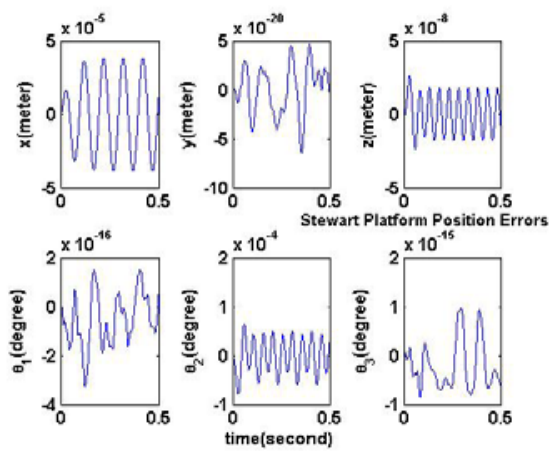

Figure 10: Stewart platform position errors
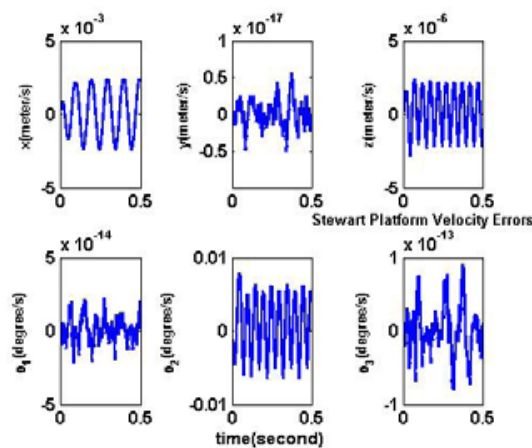

Figure 11: Stewart platform velocity errors

\section{Summary}

We develop a high fidelity dynamic model for a mobile robotic system. Complete dynamic equations of motion are formulated and the dynamic interactions between the manipulator and the moving base are included. The rigorous approach we present in this chapter provides us with a tool to do high fidelity system analysis, control law verifications, and hardware in the loop emulation.

\section{References}

[1] Jeremy Davis, James Doebbler, Kevin Daugherty, John Junkins, and John Valasek. Aerospace vehicle motion emulation using omni-directional mobile platform. South Carolina, August 2007. AIAA Guidance, Navigation, and Control (GN\&C) Conference.

[2] Xaioli Bai, Jeremy Davis, James Doebbler, James Turner, and John Junkins. Dynamics and Control of the Texas A\& M Robotic Motion Emulation System. technical report TAMU-AERO-2007-07-26-1, Texas A\&M University, College Station, TX 77843-3141, July 2007.

[3] Xiaoli Bai, James D. Turner, and John L. Junkins. Dynamic analysis and control of a stewart platform

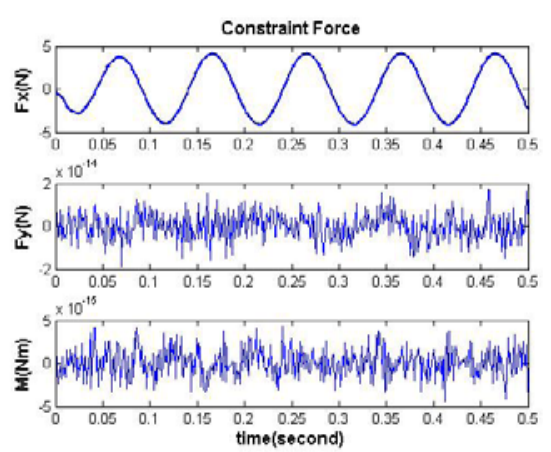

Figure 12: Constraint force/momentum

using a novel automatic differentiation method. Keystone, CO., Aug 2006. AIAA/AAS Astrodynamics Specialist Conference.

[4] Xiaoli Bai, James D. Turner, and John L. Junkins. Automatic differentiation based dynamic model for a mobile stewart platform. Greenwich, London, England, July 2006. 7th International Conference On Dynamics and Control of Systems and Structures in Space.

[5] Haoyong Yu, Matthew Spenko, and Steven Dubowsky. Omni-directional mobility using active split offset castors. Journal of Mechanical Design, 126(5):822-829, Sep 2004.

[6] R. Fierro and F. L. Lewis. Control of a nonholomic mobile robot: Backstepping kinematics into dynamics. Journal of Robotic Systems, 14(3):149 - 163, Dec 1997.

[7] Y. Kanayama, Y. Kimura, F. Miyazaki, and T. Noguchi. A stable tracking control method for an autonomous mobile robot. In Robotics and $A u$ tomation, Proc., IEEE International Conference on, volume 1, pages 384-389, Cincinnati, OH, May 1990.

[8] Y. Yamamoto and X. Yun. Coordinating locomotion and manipulation of a mobile manipulator. In Decision and Control, Proc. of the 31st IEEE Conference on, volume 3, pages 2643-2648, Tucson, AZ, Dec. 1992.

[9] A.M. Bloch, M. Reyhanoglu, and N.H. McClamroch. Control and stabilization of nonholonomic dynamic systems. Automatic Control, IEEE Transactions on, 37(11):1746-1757, Nov 1992.

[10] I. Kolmanovsky and N.H. McClamroch. Developments in nonholonomic control problems. Control Systems Magazine, IEEE, 15(6):20-36, Dec. 1995.

[11] J. C. Chiou and S. D. Wu. Constraint violation stabilization using inputoutput feedback linearization in 
multibody dynamic analysis. Journal of Guidance, Control, and Dynamics, 21(2):222-228, 1998.

[12] Animatics SmartMotor $\quad 2315 D T$. http://www.animatics.com.

[13] N.A.M. Hootsmans and S. Dubowsky. Large motion control of mobile manipulators including vehicle suspension characteristics. In Robotics and Automation, Proc., IEEE International Conference on, volume 3, pages 2336-2341, Sacramento, CA, April 1991.

[14] Jae H. Chung and Steven A. Velinsky. Robust interaction control of a mobile manipulator dynamic model based coordination. Journal of Intelligent and Robotic Systems, 26(1):47-63, Sep 1999.

[15] Hanspeter. Schaub and John L. Junkins. Analytical Mechanics of Space Systems. AIAA, 2003. 